\title{
Electron-neutrino scattering off nuclei from two different theoretical perspectives
}

\author{
M. Martini, ${ }^{1,2}$ N. Jachowicz, ${ }^{1}$ M. Ericson, ${ }^{3,4}$ V. Pandey, ${ }^{1}$ T. Van Cuyck, ${ }^{1}$ and N. Van Dessel ${ }^{1}$ \\ ${ }^{1}$ Department of Physics and Astronomy, Ghent University, Proeftuinstraat 86, B-9000 Gent, Belgium \\ ${ }^{2}$ ESNT, CEA-Saclay, IRFU, Service de Physique Nucléaire, F-91191 Gif-sur-Yvette Cedex, France \\ ${ }^{3}$ Université de Lyon, Université Lyon 1, CNRS/IN2P3, IPN Lyon, F-69622 Villeurbanne Cedex, France \\ ${ }^{4}$ Physics Department, Theory Unit, CERN, CH-1211 Geneva, Switzerland \\ (Received 31 January 2016; revised manuscript received 14 June 2016; published 11 July 2016)
}

\begin{abstract}
We analyze charged-current electron-neutrino cross sections on carbon. We consider two different theoretical approaches, on one hand the continuum random phase approximation (CRPA) which allows a description of giant resonances and quasielastic excitations, on the other hand the RPA-based calculations which are able to describe multinucleon emission and coherent and incoherent pion production as well as quasielastic excitations. We compare the two approaches in the genuine quasielastic channel, and find a satisfactory agreement between them at large energies while at low energies the collective giant resonances show up only in the CRPA approach. We also compare electron-neutrino cross sections with the corresponding muon-neutrino ones in order to investigate the impact of the different charged-lepton masses. Finally, restricting to the RPA-based approach, we compare the sum of quasielastic, multinucleon emission, coherent, and incoherent one-pion production cross sections (folded with the electron-neutrino T2K flux) with the charged-current inclusive electron-neutrino differential cross sections on carbon measured by T2K. We find a good agreement with the data. The multinucleon component is needed in order to reproduce the $\mathrm{T} 2 \mathrm{~K}$ electron-neutrino inclusive cross sections.
\end{abstract}

DOI: 10.1103/PhysRevC.94.015501

\section{INTRODUCTION}

Recent years have seen an accumulation of data on muonneutrino cross sections on nuclei at intermediate energies [119]. These measurements have revealed interesting features in different reaction channels. For example, the charged-current quasielastic (CCQE) measurement performed by MiniBooNE [1] has attracted a lot of attention due to its unexpected behavior, reproducible with an unphysical value of the axial mass. This axial mass anomaly is now explained by the inclusion of events in which several nucleons are ejected in the CCQE cross section [20-33]. In the one-pion production channel some questions are still open. For instance, various theoretical models $[34,35]$ cannot simultaneously reproduce the MiniBooNE [2,5] and the MINERvA [15] results.

The wealth of experimental and theoretical results on muon-neutrino cross sections contrasts with the few published results on electron-neutrino cross sections. After the inclusive $v_{e}$ CC total cross sections measured by the Gargamelle bubble chamber in 1978 [36], the first measurement of inclusive $v_{e}$ CC differential cross sections on carbon was performed by T2K [37]. Recently the measurement performed by MINERvA of quasielastic and quasielastic-like differential cross sections on carbon also appeared [38]. A precise knowledge of $v_{\mu}$ and $v_{e}$ cross sections is important in connection to the $v_{\mu} \rightarrow v_{e}$ oscillation experiments which aim at the determination of the neutrino mass hierarchy and the search for $\mathrm{CP}$ violation in the lepton sector. A theoretical comparison of the $v_{\mu}$ and $v_{e}$

Published by the American Physical Society under the terms of the Creative Commons Attribution 3.0 License. Further distribution of this work must maintain attribution to the author(s) and the published article's title, journal citation, and DOI. cross sections was performed by Day and McFarland [39] who analyzed the influence of the final lepton-mass difference on the cross sections as a function of the neutrino energy and of $Q^{2}$. Here we study these differences focusing on the $v_{\mu}$ and $v_{e}$ differential cross sections. In a first part we consider the electron-neutrino cross sections on carbon using two different theoretical models. The first one is the one of Martini et al. [20] which is based on nuclear response functions, treated in the random phase approximation (RPA) on top of a local relativistic Fermi gas (LRFG) calculation. It includes the quasielastic cross section, multinucleon emission and coherent and incoherent single pion production. The second model is the one of Jachowicz et al. [40] which is based on the continuum random phase approximation (CRPA) on top of Hartree-Fock (HF) calculations. It was originally developed to study electroweak reactions in the giant resonance region and then extended by Pandey et al. [41,42] to the quasielastic regime. The common channel where the two approaches can be compared is hence the quasielastic one. After a description of the two theoretical models, we confront their results in the quasielastic channel, first for fixed kinematics. We also illustrate in both models the differences between $v_{\mu}$ and $v_{e}$ cross sections. The difference between the two approaches manifests itself for small values of the neutrino energy $\left(E_{v} \simeq 200 \mathrm{MeV}\right)$. However when the cross sections are folded with the T2K and the MiniBooNE $v_{e}$ fluxes which are peaked at $E_{v} \simeq 500 \mathrm{MeV}$ it turns out that the differences are washed out. This justifies the use of the RPA approach of Martini et al., which incorporates, beyond the quasielastic, one pion production and multinucleon emission to compare with the inclusive $v_{e} \mathrm{CC}$ differential cross sections on carbon recently measured by $\mathrm{T} 2 \mathrm{~K}$ [37]. We postpone the comparison with the very recent MINERvA results [38] to a future paper. 


\section{THEORETICAL MODELS}

We summarize here the basic ingredients of the two models. Both approaches calculate the polarization propagator $\Pi$ in the random phase approximation (RPA) which allows the inclusion of long-range nucleon-nucleon correlations. This amounts to solving integral equations which have the generic form

$$
\Pi=\Pi^{0}+\Pi^{0} V \Pi
$$

where $\Pi^{0}$ is the bare polarization propagator and $V$ denotes the effective particle-hole interaction. However, the bare polarization propagator and the residual interaction differ in the two approaches.

For Martini et al. [20] the bare polarization propagator is evaluated in momentum space. In a finite system it is nondiagonal and writes $\Pi^{0}\left(\omega, \mathbf{q}, \mathbf{q}^{\prime}\right)$. In order to account for the finite-size effects, it is evaluated in a semiclassical approximation $[43,44]$ where it can be cast in the form

$$
\Pi^{0}\left(\omega, \mathbf{q}, \mathbf{q}^{\prime}\right)=\int d \mathbf{r} e^{-i\left(\mathbf{q}-\mathbf{q}^{\prime}\right) \cdot \mathbf{r}} \Pi^{0}\left(\omega, \frac{\mathbf{q}+\mathbf{q}^{\prime}}{2}, \mathbf{r}\right)
$$

This semiclassical calculation is a Wigner-Kirwood expansion in $\hbar$ restricted to first order. Its validity has been explored by Stroth et al. [45] and by Chanfray and Schuck [43] with the conclusion that the semiclassical description agrees with a quantum mechanical calculation but for small momenta ( $q<200 \mathrm{MeV} / c$ for light nuclei). The nondiagonality in the momenta of $\Pi^{0}\left(\omega, \mathbf{q}, \mathbf{q}^{\prime}\right)$ shows that this description goes beyond the infinite nuclear matter one. In order to obtain the quantity in the integrand of Eq. (2), a local density approximation is used which relates it to the relativistic Fermi gas polarization propagator according to

$$
\Pi^{0}\left(\omega, \frac{\mathbf{q}+\mathbf{q}^{\prime}}{2}, \mathbf{r}\right)=\Pi_{k_{F}(r)}^{0}\left(\omega, \frac{\mathbf{q}+\mathbf{q}^{\prime}}{2}\right) .
$$

The local Fermi momentum $k_{F}(r)$ is related to the experimental nuclear density through $k_{F}(r)=\left(3 / 2 \pi^{2} \rho(r)\right)^{1 / 3}$. The density profile of ${ }^{12} \mathrm{C}$ is taken from the sum-of-Gaussians nuclear charge density distribution parameters according to Ref. [46]. As for the collective RPA effects, the microscopic approach first introduced by Delorme et al. [47] is used. The method is described in detail in Ref. [20]. In the bubble RPA chain for the spin-isospin channel the possibility of $\Delta$-hole excitation is taken into account.

In the approach of Jachowicz et al. [40], the starting point is the continuum Hartree-Fock model which evaluates the bound and the continuum single-nucleon wave functions through the solution of the Schrödinger equation with a mean field potential. The bare polarization propagator, in this case the $\mathrm{HF}$ one, is then calculated in coordinate space. According to Ref. [42], the maximum multipole number to calculate the multipole operators is set to $J=16$ which turns out to be sufficient to reach the convergence of the cross sections.

The particle-hole residual interaction differs as well in the two approaches. In the Martini et al. one, the parametrization in terms of pion exchange, $\rho$ exchange, and contact Landau-
Migdal parameters is used

$$
\begin{aligned}
& V_{N N}=\left(f^{\prime}+V_{\pi}+V_{\rho}+V_{g^{\prime}}\right) \boldsymbol{\tau}_{1} \cdot \boldsymbol{\tau}_{2}, \\
& V_{N \Delta}=\left(V_{\pi}+V_{\rho}+V_{g^{\prime}}\right) \boldsymbol{\tau}_{1} \cdot \boldsymbol{T}_{2}^{\dagger}, \\
& V_{\Delta \Delta}=\left(V_{\pi}+V_{\rho}+V_{g^{\prime}}\right) \boldsymbol{T}_{1} \cdot \boldsymbol{T}_{2}^{\dagger} .
\end{aligned}
$$

For instance, in the $N N$ case one has

$$
\begin{aligned}
& V_{\pi}=\left(\frac{g_{r}}{2 M_{N}}\right)^{2} F_{\pi}^{2}\left(Q^{2}\right) \frac{\boldsymbol{q}^{2}}{\omega^{2}-\boldsymbol{q}^{2}-m_{\pi}^{2}} \sigma_{1} \cdot \widehat{q} \boldsymbol{\sigma}_{2} \cdot \widehat{q} \\
& V_{\rho}=\left(\frac{g_{r}}{2 M_{N}}\right)^{2} C_{\rho} F_{\rho}^{2}\left(Q^{2}\right) \frac{\boldsymbol{q}^{2}}{\omega^{2}-\boldsymbol{q}^{2}-m_{\rho}^{2}} \sigma_{1} \times \widehat{q} \sigma_{2} \times \widehat{q} \\
& V_{g^{\prime}}=\left(\frac{g_{r}}{2 M_{N}}\right)^{2} F_{\pi}^{2}\left(Q^{2}\right) g^{\prime} \sigma_{1} \cdot \sigma_{2}
\end{aligned}
$$

where $g^{\prime}$ is the Landau-Migdal parameter and $C_{\rho}=$ 1.5. Here $F_{\pi}\left(Q^{2}\right)=\left(\Lambda_{\pi}^{2}-m_{\pi}^{2}\right) /\left(\Lambda_{\pi}^{2}+Q^{2}\right)$ and $F_{\rho}\left(Q^{2}\right)=$ $\left(\Lambda_{\rho}^{2}-m_{\rho}^{2}\right) /\left(\Lambda_{\rho}^{2}+Q^{2}\right)$ are the pion-nucleon and $\rho$-nucleon form factors, with $\Lambda_{\pi}=1 \mathrm{GeV}$ and $\Lambda_{\rho}=1.5 \mathrm{GeV}$. Their presence suppresses the residual forces at large $Q^{2}$. For the Landau-Migdal parameter $f^{\prime}$ the value $f^{\prime}=0.6$ is taken. As for the spin-isospin parameters $g^{\prime}$ the following values are used: $g_{N N}^{\prime}=0.7, g_{N \Delta}^{\prime}=g_{\Delta \Delta}^{\prime}=0.5$.

In the Jachowicz et al. model, the zero-range Skyrme effective interaction, in its parametrization called SkE2 [48,49], is used. The same interaction which enters in the mean field calculation is employed to generate the continuum RPA (CRPA) solution. In this way, the calculation becomes self-consistent with respect to the interaction. In Ref. [42] this residual Skyrme-type interaction is multiplied by a dipole form factor which controls the influence of the residual interaction at high $Q^{2}$ values [50]. At variance with respect to Ref. [42], here the CRPA results are not folded with a Lorentz function which would spread the strength of giant resonances without modification of the position of the quasielastic peak.

Concerning the RPA differences, an important point should be mentioned. The possibility of $\Delta$-hole excitation in the RPA chain is included explicitly in the case of Martini et al. This is reflected in the appearance in certain kinematical regions of a sizable quenching in the RPA results, due to the mixing of nucleon-hole states with $\Delta$-hole ones, the Ericson-EricsonLorentz-Lorenz (EELL) effect [51]. This quenching has been introduced and established in pion scattering [51]. It has been discussed also in relation with electron [52] and neutrino $[20,25,53]$ scattering.

\section{COMPARISON BETWEEN THEORETICAL CALCULATIONS}

\section{A. LRFG+RPA vs HF+CRPA}

In this subsection, we compare the theoretical results in the one nucleon-one hole sector obtained in the two different approaches. We consider the $v_{e^{-}}{ }^{12} \mathrm{C}$ double differential cross sections for different values of the neutrino energy and lepton scattering angle. These cross sections are purely theoretical quantities since the experimental ones depend on the neutrino fluxes and hence are specific for each experiment. In Fig. 1 we 


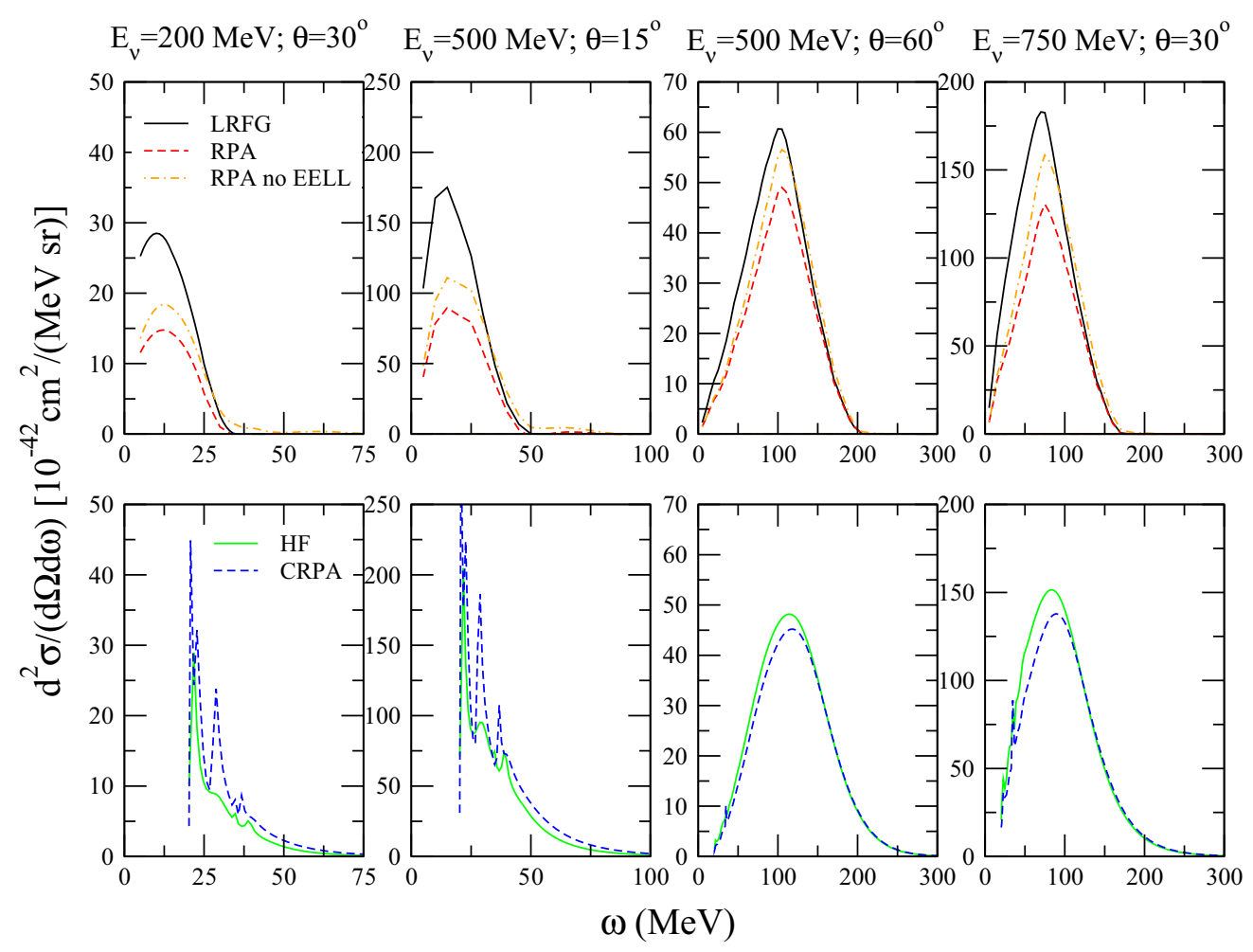

FIG. 1. Electron-neutrino CC double differential cross section on carbon for fixed values of scattering angles and incident neutrino energies as a function of the energy transferred to the nucleus. In the upper panels the results obtained in the bare-LRFG approach and in the RPA with and without the EELL effect are displayed. In the lower panels the results obtained in HF and CRPA approaches are displayed. Only genuine quasielastic and giant resonance excitations (given by the CRPA) are considered.

display the results of the two approaches by switching on and off the residual particle-hole interaction. We keep the same notations as in the previous papers of the groups. Namely we call "bare-LRFG" the results of Martini et al. when the particle-hole interaction is switched off (these are relativistic Fermi gas results in the local density approximation) and "RPA" the results obtained by switching on the particle-hole interaction. The corresponding results in the case of Jachowicz et al. are called "HF" and "CRPA". Some important differences between the two approaches appear. The most striking feature is the appearance of giant resonance peaks in the CRPA results of Jachowicz et al. They vanish for large neutrino energy or larger scattering angle. The second comment concerns the threshold energy in the HF+CRPA approach, about $\simeq 18 \mathrm{MeV}$, which reflects the nucleon separation energy, ignored in the semi-classical approximation of Martini et al. The HF+CRPA results also display the shell structure, which is not present in the semiclassical description. It disappears at large angles or energies, where the two approaches become similar. However, when compared to the semiclassical LRFG results, in the mean field HF case the quasielastic peak is somewhat quenched and the high transferred-energy tail is more important. As discussed in Ref. [45] this is a consequence of the nonlocality of the mean field which has a similar effect as the residual transverse repulsive ph interaction: it quenches and hardens the responses.

Turning to RPA effects, the important difference is the large RPA quenching in the Martini et al. approach, due to the mixing with $\Delta$-hole states that we have commented before, not explicitly present in the CRPA results of Jachowicz et al. For completeness the RPA results without the $\Delta$-hole mixing (the EELL effect) are also shown in Fig. 1. In this context further comments are in order. The particle-hole force which enters in the RPA chain, as given in Eqs. (4), (5), decreases with the momentum transfer. This is apparent in the upper panels of Fig. 1 from the differences between the bare (LRFG) and RPA cases with or without the EELL effect. Notice also that the influence of the RPA in the approach of Jachowicz et al. in the larger momentum cases, even if small, is similar to the one of the Martini et al. without the EELL effect: it leads to a small quenching and hardening of the cross section. This behavior is less evident at low momentum transfer, where the main difference between HF and CRPA is in the appearance of the giant resonances, however one can notice a hardening of the strength in the CRPA tails.

In order to better illustrate the comparison between the two approaches, we show in Fig. 2 the LRFG and RPA results shifted by $18 \mathrm{MeV}$, an average value of the separation energy, and we compare them with the HF and CRPA results, respectively. For the structureless part of the cross sections, i.e., for the kinematical conditions such as $\theta=60^{\circ}$ and $E_{v_{e}}=500 \mathrm{MeV}$ or $\theta=30^{\circ}$ and $E_{v_{e}}=750 \mathrm{MeV}$, the two approaches are essentially in agreement. Furthermore the HF or CRPA cross sections are characterized by a stronger tail at high transferred energies, an effect, as mentioned before, of the nonlocality of the mean field. It turns out that, in 


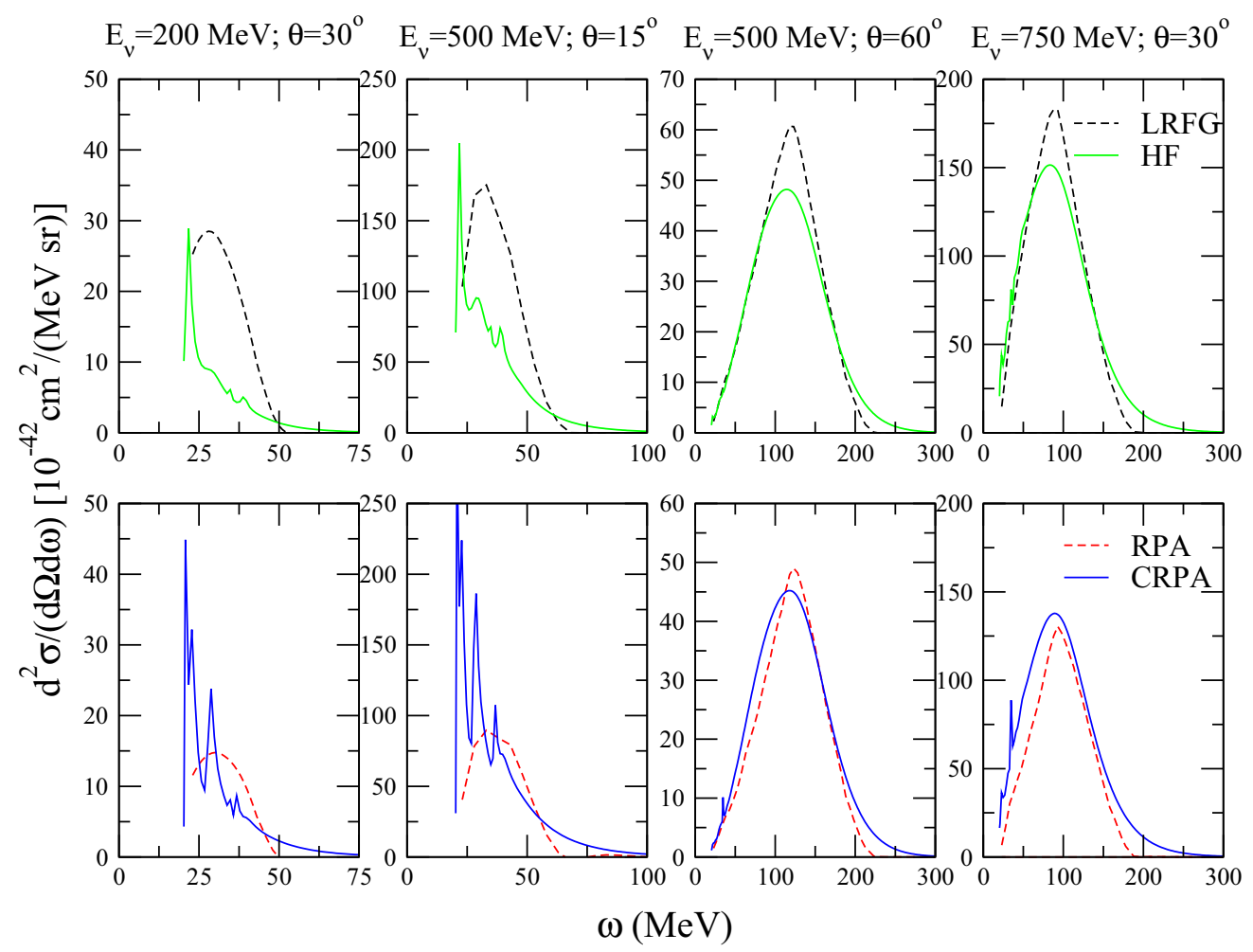

FIG. 2. Electron-neutrino CC double differential cross section on carbon for fixed values of scattering angles and incident neutrino energies as a function of the energy transferred to the nucleus. In the upper (lower) panels the results obtained in the bare-LRFG (RPA) and HF (CRPA) approaches are displayed. Only genuine quasielastic and giant resonances excitations (given by the CRPA) are considered. Continuous lines: HF and CRPA results; dashed lines: LRFG and RPA results shifted by $18 \mathrm{MeV}$.

the low-energy part, the RPA results (which do not show giant resonance peaks) represent the average of the CRPA calculations relatively well, in spite of the fact that the semi-classical approach is not expected to be valid in this region, which corresponds to low transferred momenta.

Another interesting information concerns the main multipoles contributing to the CRPA cross sections, in particular for the resonant structures. To illustrate this point we plot in Fig. 3 the three major multipole contributions to the cross sections in the four kinematical conditions of Figs. 1 and 2. As it clearly appears, at low neutrino energy and forward scattering angles, the cross section, with its giant resonance structures is dominated by the $1^{-}, 2^{-}$, and $2^{+}$ multipole contributions. On the contrary at larger energies and scattering angles the contribution of the various multipoles is more evenly distributed, as discussed in Ref. [40]. One can appreciate this point by noticing that the first three major multipole contributions are of the same order of magnitude and are not dominant, hence one needs to include all multipole contributions up to $J=16$ with natural and unnatural parity.

Turning to the flux folded cross sections we consider the T2K [54] and MiniBooNE [55] $v_{e}$ normalized fluxes, which are shown in Fig. 4. We discuss single-differential quasielastic cross sections, $\frac{d \sigma}{d p_{e}}$ and $\frac{d \sigma}{d \cos \theta_{e}}$, their theoretical evaluation is displayed in Fig. 5. One observes that the giant resonance

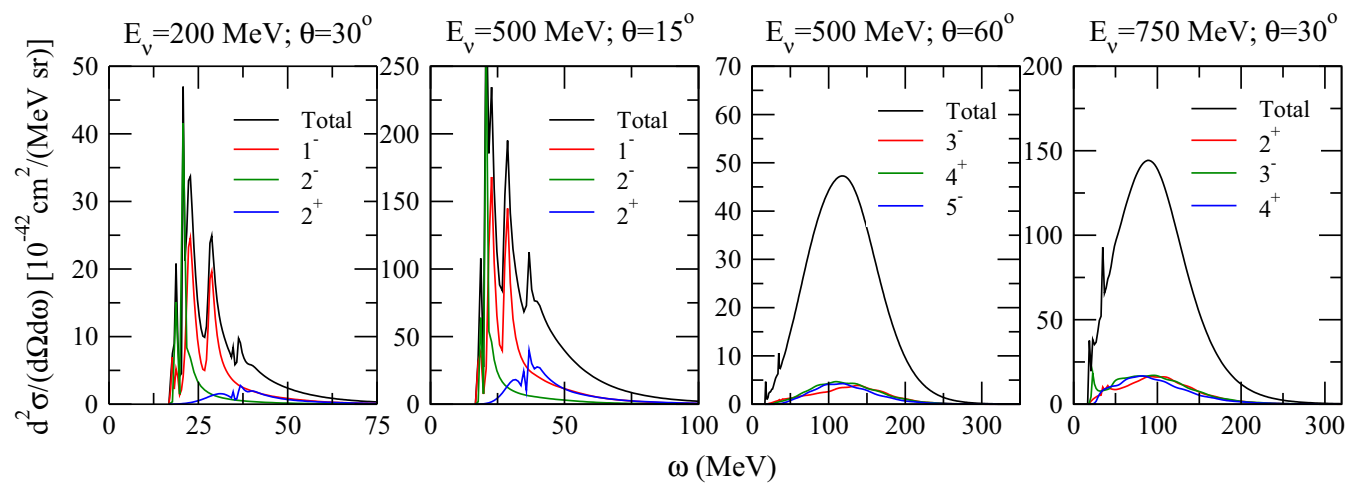

FIG. 3. The three major multipole contributions to the CRPA cross sections in the different kinematical conditions. 


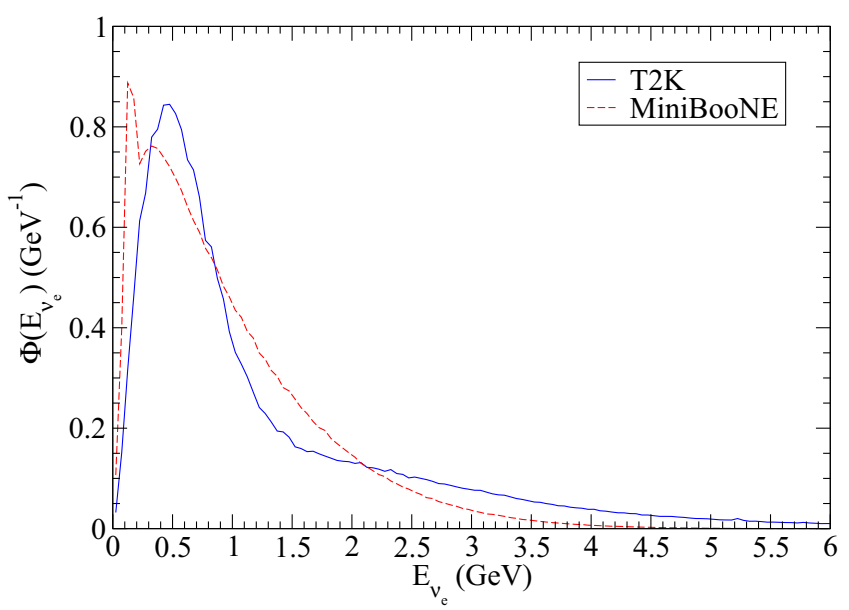

FIG. 4. Normalized electron-neutrino T2K [54] and MiniBooNE [55] fluxes.

effects are no longer apparent in the CRPA and that in general the differences between HF and CRPA calculations are largely washed out by the flux folding. Moreover in the case of MiniBooNE fluxes the HF or CRPA results are very similar to the LRFG ones while the RPA curves, which are somewhat below, display the usual EELL quenching. The results without this EELL quenching are also shown in Fig. 5. In the T2K case instead some small differences appear: the HF and CRPA results are above the corresponding LRFG cross section. This difference, which did not show with the MiniBooNE flux, is the effect of the larger T2K high energy tail. The differences between the two different theoretical models are weighted in different ways by the different flux profiles. But in general the differences between the various models are small and beyond the present level of experimental accuracy.

\section{B. $v_{e}$ vs $v_{\mu}$ cross sections}

After the discussion of the differences between the two approaches for the $v_{e}$ case, we turn to a comparison between the charged current $v_{e}$ and $v_{\mu}$ cross sections. We perform this comparison for different final state channels. For the sake of illustration, we present in Fig. 6 the double differential cross sections in different channels (genuine quasielastic, multinucleon excitations, and one-pion production) for fixed values of the scattering angle (60 degrees) and neutrino energy $\left(E_{v}=500 \mathrm{MeV}\right)$ as a function of the lepton kinetic energy $T_{l}$, a measurable quantity. The role of the different charged lepton masses appears not only in the trivial relative shift between the $v_{e}$ and $v_{\mu}$ CC cross sections, according to the identities

$$
T_{l}=E_{l}-m_{\text {lepton }}=E_{v}-\omega-m_{\text {lepton }}=\omega_{\max }-\omega,
$$

but also in the strength and in the shape of the cross sections, particularly in the pion production channel. In order to eliminate trivial mass shift effects we plot in the following figures (Figs. 7, 9, and 11) the differential cross sections as a function of the energy transfer $\omega=E_{v}-m_{\text {lepton }}-T_{l}$.

We start with the CRPA case which allows a simultaneous treatment of giant resonances and quasielastic excitations. In Fig. 7, we display the double-differential cross sections for different values of incoming neutrino energy and lepton scattering angle, both for $v_{e}$ and $v_{\mu}$. In most cases the $v_{e}$ and $v_{\mu}$ results are quite similar, sometimes practically indistinguishable. However, in some cases interesting differences appear. The first one is a consequence of the stringent limit on the maximum transferred energy $\omega_{\max }=E_{v}-m_{\text {lepton }}$ which has smaller values in the muon case. This threshold effect can
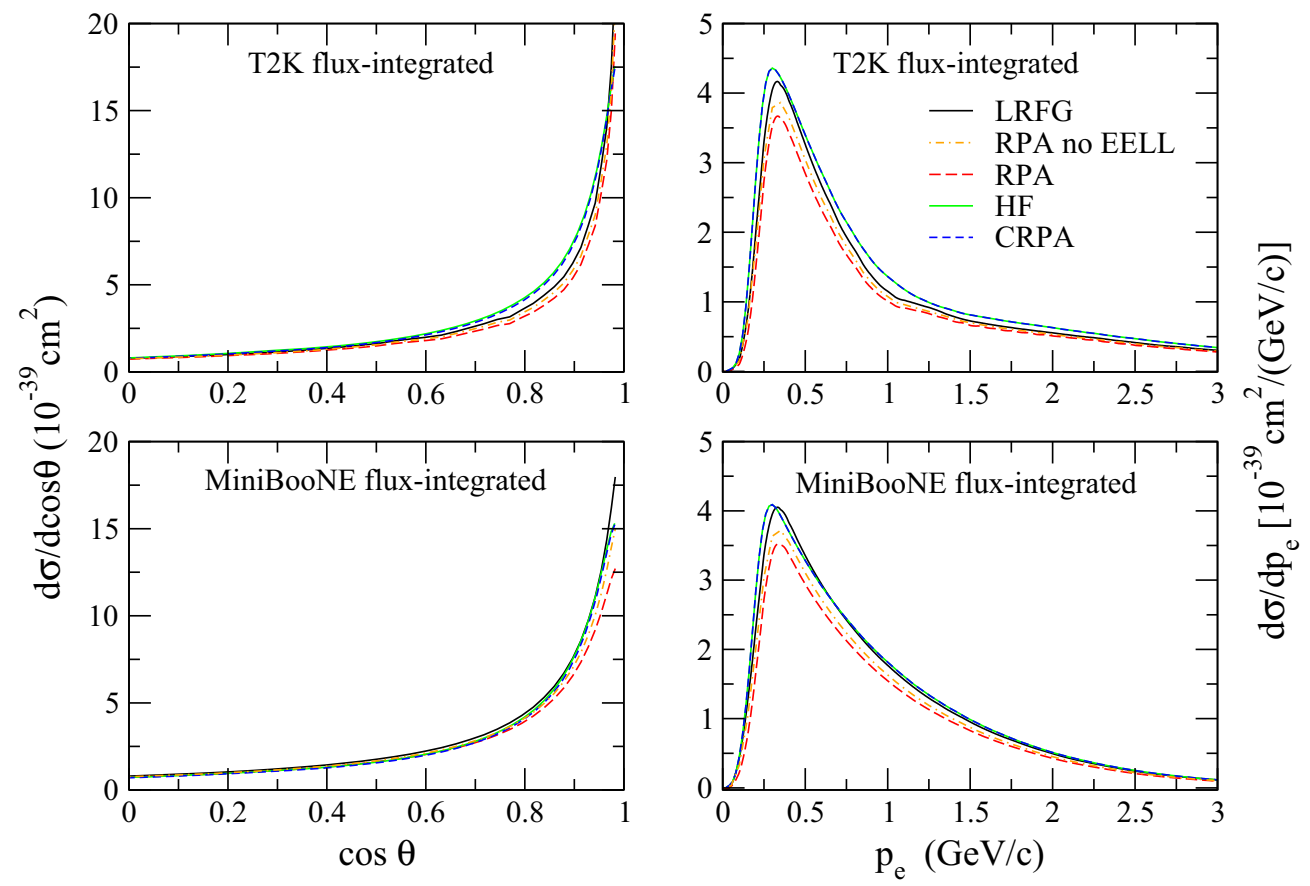

FIG. 5. Electron-neutrino T2K and MiniBooNE flux-folded CC single differential cross sections on carbon per nucleon. 

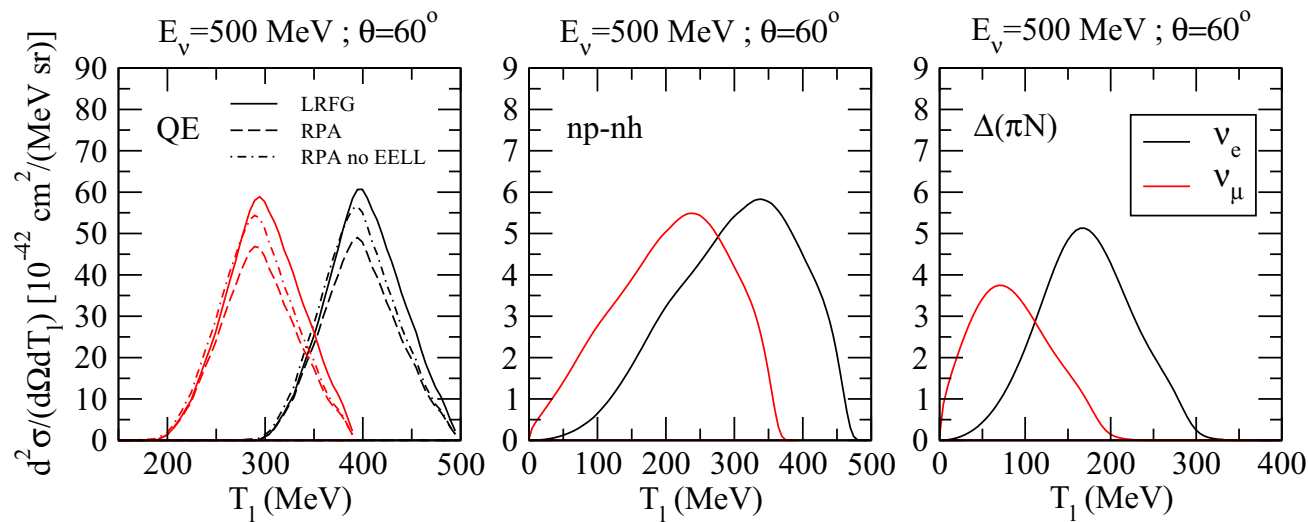

FIG. 6. Electron- and muon-neutrino CC double differential cross section on carbon for fixed values of scattering angle and incident neutrino energies as a function of the charged lepton kinetic energy calculated in the RPA approach of Ref. [20]. Left panel: genuine quasielastic with and without RPA and EELL effects; middle panel: np-nh excitations; right panel: incoherent one pion production contribution.

be observed in Fig. 7 for $E_{v}=150 \mathrm{MeV}$ and for $E_{v}=200$ $\mathrm{MeV}$ in the case of 60 degrees. Other differences can be appreciated by observing the evolution with the scattering angle of the cross sections at small neutrino energies such as $E_{v}=150 \mathrm{MeV}$ or $E_{v}=200 \mathrm{MeV}$. For small scattering angles

such as 5 degrees, $v_{\mu}$ cross sections are higher than the $v_{e}$ ones, while for larger scattering angles, for example 60 degrees this behavior is opposite. At intermediate angles the two cross sections are closer to each other. This angular behavior weakly survives at $E_{v}=500 \mathrm{MeV}$ while for $E_{v}=750 \mathrm{MeV}$ the $v_{e}$

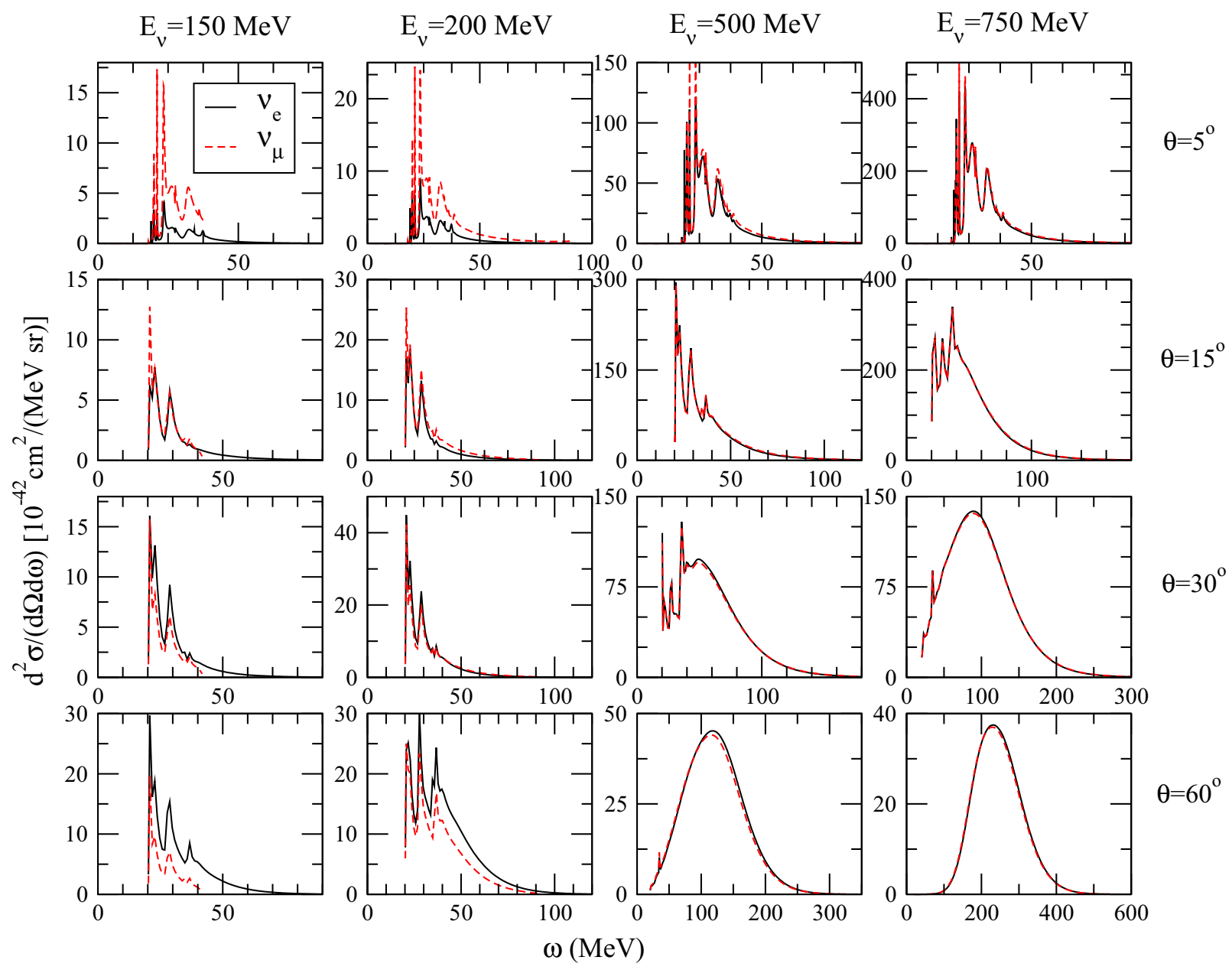

FIG. 7. Electron- and muon-neutrino CC double differential cross section on carbon calculated in the CRPA approach for fixed values of scattering angles and incident neutrino energies as a function of the energy transferred to the nucleus. 


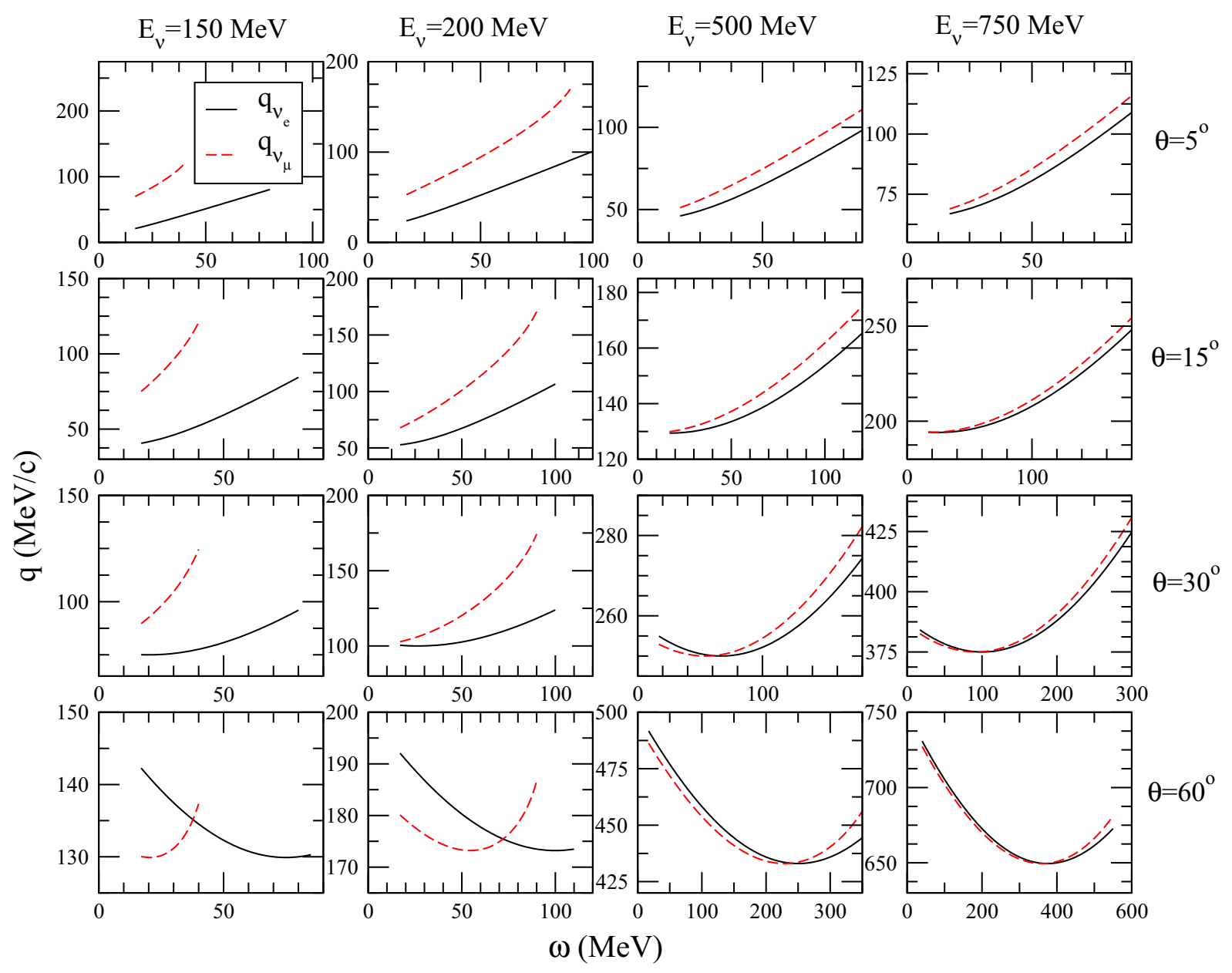

FIG. 8. The values of the momentum transfer versus the transferred energy for the $v_{e}$ and $v_{\mu}$ scattering corresponding to the kinematical conditions of Fig. 7.

and $v_{\mu}$ cross sections practically coincide for all the scattering angles. The differences discussed above are also related to the differences in the momentum transfer between the $v_{e}$ and $v_{\mu}$ scattering. For completeness we show in Fig. 8 the values of the momentum transfer spanned in the 16 panels of Fig. 7. As expected, the major differences between $v_{e}$ and $v_{\mu}$ appear at small neutrino energies where threshold effects are more evident. Furthermore at small scattering angle the momentum transfer is always larger for $v_{\mu}$ than for $v_{e}$ while at 60 degrees this is not always the case.

It is also interesting to illustrate the behavior of $v_{e}$ and $v_{\mu}$ cross sections by separating their contributions, as shown in Fig. 9 for incoming neutrino energies of $E_{v}=200 \mathrm{MeV}$ and $E_{v}=750 \mathrm{MeV}$. According to the notation of Ref. [41], the global contribution related to the Coulomb and longitudinal multipole excitation operators (containing vector and axial components) is labeled as CL. In the language of Refs. [20,21] it represents the sum of isovector and isospin spin-longitudinal response contributions. The sum of transverse contributions, including the vector-axial interference term, is labeled as $\mathrm{T}$. These are the terms containing the isospin spin-transverse response in the language of Refs. [20,21]. As one can observe in Fig. 9, for $E_{v}=200 \mathrm{MeV}$ and $\theta=5$ degrees (and in general for very forward scattering) the neutrino cross section is dominated by the CL contribution while for larger angles, such as 60 degrees, the transverse contribution $\mathrm{T}$ is dominant. At larger energies the transverse part dominates everywhere except for very small scattering angles. At $E_{v}=200 \mathrm{MeV}$ and $\theta=5$ degrees the dominant CL contribution to the cross sections, as well as the smaller $\mathrm{T}$ one, are larger for $v_{\mu}$ than for $v_{e}$, hence the larger $v_{\mu}$ cross sections for this case. The relative weight of CL and T contributions is the result of a subtle interplay between lepton kinematic factors and response functions. The competition for dominance of the cross section between both, is very sensitive to energy and momentum transfer. The surprising dominance of $v_{\mu}$ over $v_{e}$ cross sections for small scattering angles is related to this and dictated by the non-trivial dependence of momentum transfer on lepton mass and scattering angle for forward scattering, as illustrated in Fig. 8.

The nontrivial behavior of the $v_{e}$ cross sections with respect to the $v_{\mu}$ ones is also illustrated in Fig. 10 where the ratio of the single differential cross section $\frac{d \sigma_{v_{e}}}{d \cos \theta} / \frac{d \sigma_{v_{\mu}}}{d \cos \theta}$ is shown for $E_{v}=200 \mathrm{MeV}$ and $E_{v}=750 \mathrm{MeV}$. In the low energy case of $E_{v}=200 \mathrm{MeV}$ where the semiclassical description breaks down, we restrict ourself to the CRPA approach. In this case the 

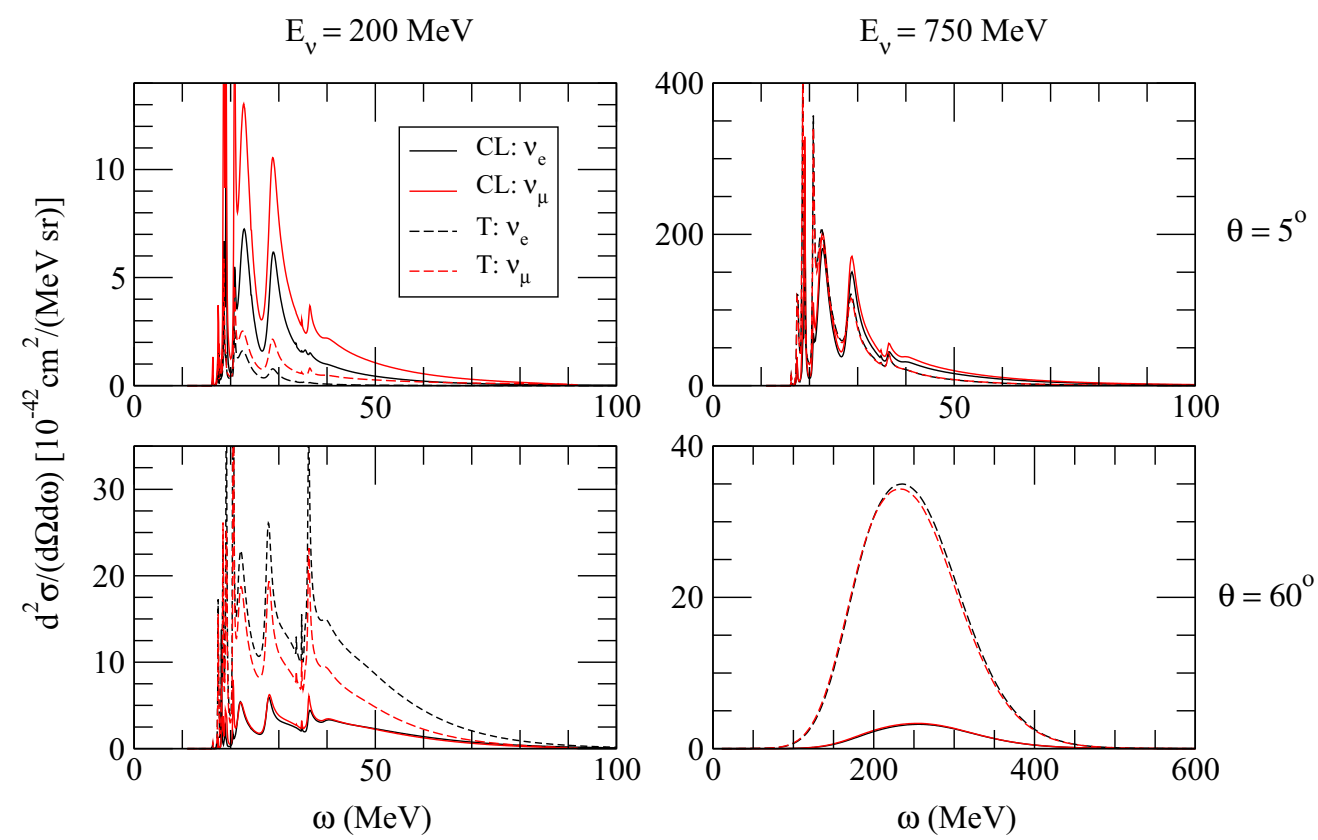

FIG. 9. Coulomb-longitudinal (CL) and transverse (T) contributions to electron- and muon-neutrino CC double differential cross section on carbon calculated in the CRPA approach for incident neutrino energies of $200 \mathrm{MeV}$ and $750 \mathrm{MeV}$ and two fixed values of scattering angles as a function of the transferred energy to the nucleus.

ratio deviates very appreciably from 1 while at larger neutrino energies, such as $E_{v}=750 \mathrm{MeV}$, it gets closer to 1 in the CRPA as well as in the RPA case with or without the EELL effect. In Fig. 10 this quantity $\frac{d \sigma_{v_{e}}}{d \cos \theta} / \frac{d \sigma_{\nu}}{d \cos \theta}$ at $E_{v}=750 \mathrm{MeV}$ is given also for two other channels, the pion production and multinucleon excitations in the RPA approach of Martini et al. where they are available. This $\frac{d \sigma_{v_{e}}}{d \cos \theta} / \frac{d \sigma_{v_{\mu}}}{d \cos \theta}$ ratio, always larger than 1 , is characterized by a smooth decreasing behavior. For the pion emission channel (via $\Delta$ excitation) this ratio is larger than the one for the np-nh and $1 \mathrm{p}-1 \mathrm{~h}$ excitations.
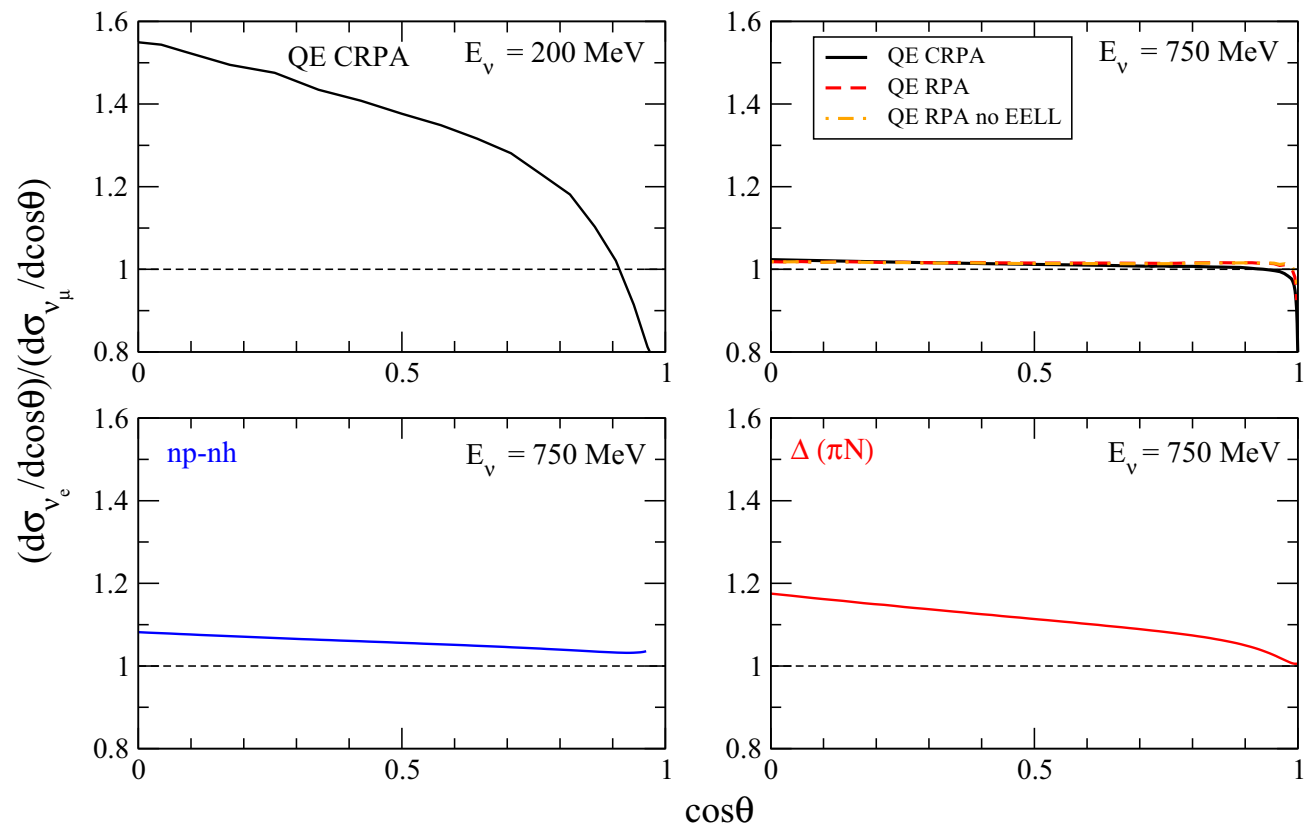

FIG. 10. Ratio of the $v_{e}$ over $v_{\mu}$ differential cross section on carbon calculated for two fixed values of incident neutrino energies as a function of the cosine of the lepton scattering angle. The $1 \mathrm{p}-1 \mathrm{~h}$ results in the CRPA approach are shown for $E_{v}=200 \mathrm{MeV}$ and $E_{v}=750 \mathrm{MeV}$. The $1 \mathrm{p}-1 \mathrm{~h}$ results in the RPA approach with or without the EELL effect, the $n \mathrm{p}-n \mathrm{~h}$ excitations and the one-pion production (via $\Delta$ excitation) results are shown for $E_{v}=750 \mathrm{MeV}$. 

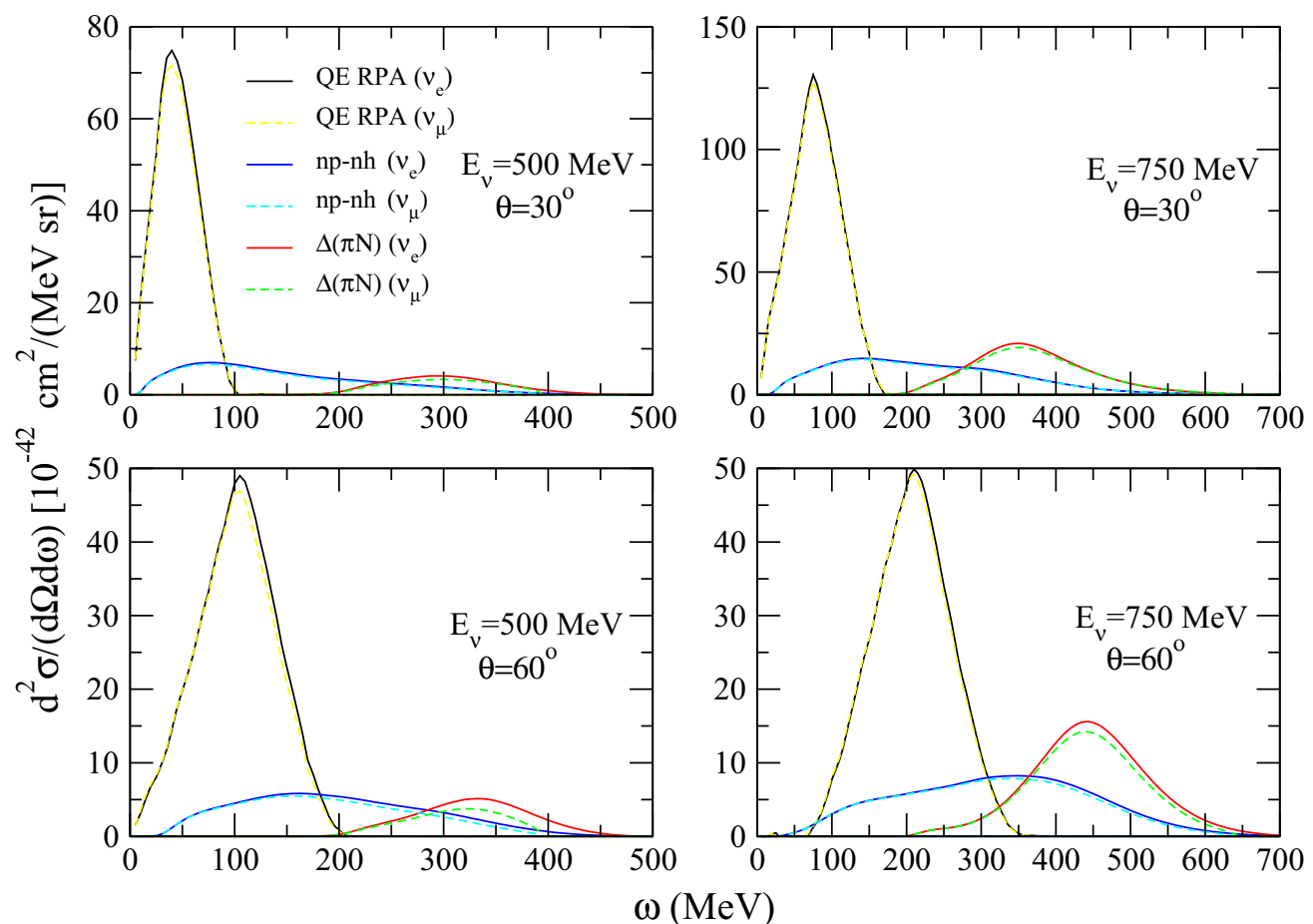

FIG. 11. Electron- and muon-neutrino CC double differential cross section on carbon calculated in the RPA approach of Martini et al. for fixed values of scattering angles and incident neutrino energies as a function of the transferred energy to the nucleus. The genuine quasielastic (QE), multinucleon (np-nh), and incoherent one-pion production excitations are plotted separately.

Concerning the pion production and multinucleon excitations, we display for completeness in Fig. 11 the $v_{e}$ and $v_{\mu}$ results obtained for these channels (as well as for the QE one) in the RPA approach of Martini et al. for the double differential cross sections at incoming neutrino energies of $E_{v}=500 \mathrm{MeV}$ and $E_{v}=750 \mathrm{MeV}$ and scattering angles of 30 and 60 degrees. One observes the clear energy separation between the three channels, the highest energy transfer occurring for pion emission. Ignoring Fermi momentum and RPA reshaping effects, the quasielastic peak occurs for an energy transfer $\omega=Q^{2} /\left(2 M_{N}\right)$, where $Q^{2}=$ $q^{2}-\omega^{2}=2 E_{v} E_{l}(1-\cos \theta)-m_{l}^{2}+2 E_{v}\left(E_{l}-P_{l}\right) \cos \theta$. In the electron case where $m_{l}=0$ it leads to $\omega=E_{v}{ }^{2}(1-$ $\cos \theta) /\left(M_{N}+E_{\nu}(1-\cos \theta)\right)$. As for pion emission, in our model it occurs via $\Delta$ excitation. In the same (nucleons at rest) approximation the pion emission peak is shifted towards large energy transfer, with the condition $\omega=Q^{2} /\left(2 M_{N}\right)+$ $\Delta M$ with $\Delta M=\left(M_{\Delta}^{2}-M_{N}^{2}\right) / 2 M_{N}=338 \mathrm{MeV}$. For $v_{e}$ this leads to $\omega=\left(M_{N} \Delta M+E_{v}{ }^{2}(1-\cos \theta)\right) /\left(M_{N}+E_{v}(1-\right.$ $\cos \theta)$ ). These formulas explain the positions of the quasielastic and $\Delta$ peaks. As for the multinucleon excitations they lie between the two. The difference between the $v_{e}$ and $v_{\mu}$ cross sections mostly shows up in the energy transfer limit which is $\omega_{\max } \simeq E_{v}$ for electrons and $\omega_{\max }=E_{v}-m_{\mu}$ for muons. Hence it shows up mostly for pion production and it is more pronounced at low neutrino energies. It is also more pronounced at large scattering angles since the double differential cross sections move towards larger $\omega$ when the scattering angle increases. This behavior with the scattering angle appears also in Fig. 10.

\section{COMPARISON WITH THE T2K $v_{e}$ INCLUSIVE CROSS SECTIONS}

The T2K collaboration published the first results for $v_{e}$ charged-current inclusive differential cross sections on carbon [37]. In this section we compare these experimental results with our predictions, restricting to the Martini et al. [20] RPA approach which describes all final channels. We compute the $v_{e}$ T2K flux integrated differential cross sections $\frac{d \sigma}{d p_{e}}$ and $\frac{d \sigma}{d \cos \theta_{e}}$ in the different excitation channels, namely quasielastic, multinucleon excitations (np-nh) and one-pion (coherent and incoherent) production. The $v_{e} \mathrm{~T} 2 \mathrm{~K}$ flux averaging favors large $E_{v}$ values which ensures the validity of the semiclassical RPA approach of Martini et al. In Figs. 12 and 13 we plot the different exclusive channel contributions separately, as well as their sum. This sum is in a good agreement with the experiment. Notice that this agreement needs the presence of the np-nh contribution (which even dominates the genuine $\mathrm{QE}$ one for small $p_{e}$ values, $p_{e} \lesssim 0.2 \mathrm{GeV}$ ), a conclusion already reached by Martini and Ericson [31] in connection with the T2K inclusive $v_{\mu}$ double differential cross sections [9]. This agreement with both $v_{\mu}$ and $v_{e} \mathrm{CC}$ inclusive T2K flux folded differential cross sections is not systematically obtained in other approaches. For instance the SuSAv2 model by Ivanov et al. [56] reproduces well the CC inclusive T2K flux folded $v_{\mu}$ double differential cross section but underestimates the CC inclusive T2K flux folded $v_{e}$ single differential cross section. A comparison with these quantities has also been performed by Meucci and Giusti using the Relativistic Green's function model which turned to underestimate the $v_{\mu}$ and $v_{e}$ $\mathrm{CC}$ inclusive T2K data [57]. 


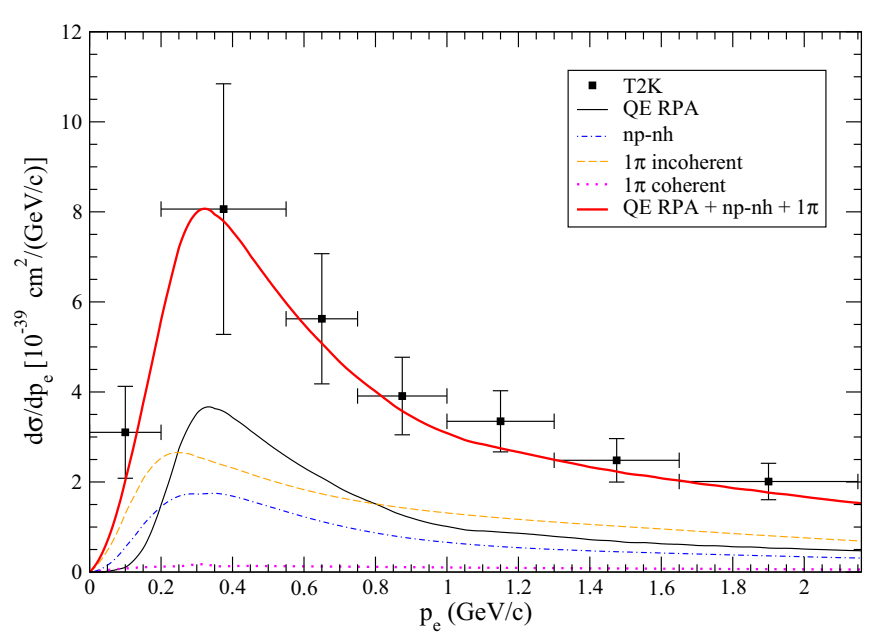

FIG. 12. T2K flux-integrated inclusive $v_{e} \mathrm{CC}$ differential cross section on carbon per nucleon as a function of the electron momentum. The different contributions to this inclusive cross section obtained in the Martini et al. RPA model of Ref. [20] are shown. The experimental T2K points are taken from Ref. [37].

\section{SUMMARY AND CONCLUSIONS}

In conclusion, our study has dealt with several facets of the neutrino interaction with nuclei. A large part is devoted to the comparison between two different approaches to describe the interaction of neutrinos with nuclei. Both go beyond the impulse approximation and take into account, albeit in different ways, the interaction between nucleons. The CRPA approach of Jachowicz et al. starts from a continuum Hartree Fock description with Skyrme type interactions. The shell structure of the nucleus is present in this approach. The RPA-based approach of Martini et al. instead starts from a semiclassical description of the bare polarization propagator with a realistic nuclear density distribution. The shell structure

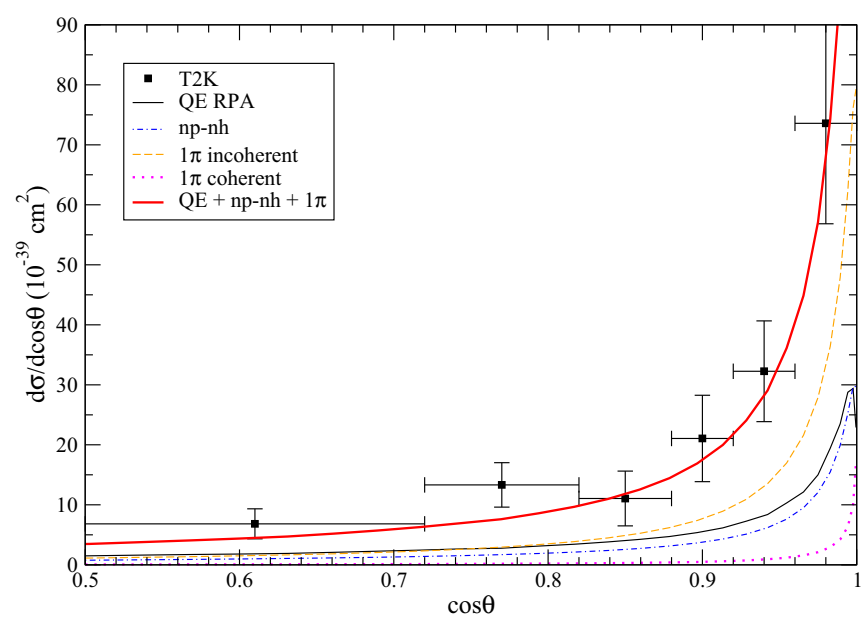

FIG. 13. T2K flux-integrated inclusive $v_{e} \mathrm{CC}$ differential cross section on carbon per nucleon as a function of the cosine of the lepton scattering angle. The different contributions to this inclusive cross section obtained in the Martini et al. RPA model of Ref. [20] are shown. The experimental T2K points are taken from Ref. [37]. is ignored in this description. The RPA effects also differ in the two approaches. For the residual interaction the first method uses the same Skyrme interaction as for the mean field, while in the approach of Martini et al., it is parametrized in terms of pion and rho exchange and a contact Landau Migdal interaction. But the main difference is the possibility of mixing of $\Delta$-hole states in the second approach. It produces a general quenching of the responses which shows up in most kinematical conditions that we have explored. The CRPA of Jachowicz et al. allows a description of giant resonances and quasielastic excitations while the RPA evaluations of Martini et al. includes quasielastic but also coherent and incoherent pion production, and multinucleon excitations.

We have compared the two approaches for the one-nucleonone-hole excitations finding a reasonable agreement between them in the quasielastic peak region, with a trend for the RPA approach to lead to lower cross sections than the CRPA presumably due to the mixing with $\Delta$ excitations. Other general trends are related to the more important high transferred-energy tail in the CRPA results and to a relative shift of the cross sections of $\omega \simeq 18 \mathrm{MeV}$, reflecting the presence of the nucleon separation energy in the CRPA calculations. The most striking difference is the appearance of giant resonance peaks in the CRPA results. The comparison of the two approaches has been performed for fixed values of the incoming neutrino energy as well as for the $v_{e} \mathrm{~T} 2 \mathrm{~K}$ and MiniBooNE flux-folded cross sections.

We have also compared the $v_{e}$ cross sections with the corresponding $v_{\mu}$ ones for fixed values of the neutrino energy in order to investigate the impact of different charged lepton masses. We have found some nontrivial behavior, in particular for the $1 \mathrm{p}-1 \mathrm{~h}$ excitations at low neutrino energies, such as an inversion with the scattering angle of the relative strength of $v_{e}$ and $v_{\mu}$ cross sections. Due to the different kinematical limits, the $v_{e}$ cross sections are in general expected to be larger than the $v_{\mu}$ ones, however for forward scattering angles this hierarchy is opposite. In the precision era of neutrino oscillation physics the $v_{e}$ cross sections should be known with the same accuracy as the $v_{\mu}$ ones. Trying to deduce the $v_{e}$ cross sections from the experimental $v_{\mu}$ ones can be considered only as a first approximation in the study of the $v_{e}$ interactions.

Concerning the comparison with experiment, we have considered the inclusive $v_{e}$ T2K flux-folded single-differential cross sections on carbon where the inclusion of np-nh and pion production effects beyond the genuine quasielastic is mandatory. For this reason we have compared the data with the only RPA-based approach of Martini et al. which includes all these channels. We have found a good agreement with the data. This success obtained with a new flux, the $v_{e} \mathrm{~T} 2 \mathrm{~K}$ one, complements those already reached with the three different fluxes, such as the MiniBooNE $v_{\mu}$ and $\bar{v}_{\mu}$, and T2K $v_{\mu}$ ones.

\section{ACKNOWLEDGMENTS}

This work was supported by the Interuniversity Attraction Poles Programme initiated by the Belgian Science Policy Office (BriX network P7/12) and by the Research Foundation Flanders (FWO-Flanders). M.M. acknowledges also the 
support and the framework of the "Espace de Structure et de réactions Nucléaire Théorique" (ESNT, http://esnt.cea.fr) at
CEA. We thank Raúl González-Jiménez, Carlotta Giusti, and Andrea Meucci for useful discussions.
[1] A. A. Aguilar-Arevalo et al. (MiniBooNE Collaboration), Phys. Rev. D 81, 092005 (2010).

[2] A. A. Aguilar-Arevalo et al. (MiniBooNE Collaboration), Phys. Rev. D 81, 013005 (2010).

[3] A. A. Aguilar-Arevalo et al. (MiniBooNE Collaboration), Phys. Rev. D 82, 092005 (2010).

[4] Y. Nakajima et al. (SciBooNE Collaboration), Phys. Rev. D 83, 012005 (2011).

[5] A. A. Aguilar-Arevalo et al. (MiniBooNE Collaboration), Phys. Rev. D 83, 052007 (2011).

[6] A. A. Aguilar-Arevalo et al. (MiniBooNE Collaboration), Phys. Rev. D 83, 052009 (2011).

[7] C. Anderson et al. (ArgoNeuT Collaboration), Phys. Rev. Lett. 108, 161802 (2012).

[8] A. A. Aguilar-Arevalo et al. (MiniBooNE Collaboration), Phys. Rev. D 88, 032001 (2013).

[9] K. Abe et al. (T2K Collaboration), Phys. Rev. D 87, 092003 (2013).

[10] G. A. Fiorentini et al. (MINERvA Collaboration), Phys. Rev. Lett. 111, 022502 (2013).

[11] L. Fields et al. (MINERvA Collaboration), Phys. Rev. Lett. 111, 022501 (2013).

[12] R. Acciarri et al. (The ArgoNeuT Collaboration), Phys. Rev. D 89, 112003 (2014).

[13] K. Abe et al. (T2K Collaboration), Phys. Rev. D 90, 052010 (2014).

[14] A. Higuera et al. (MINERvA Collaboration), Phys. Rev. Lett. 113, 261802 (2014).

[15] B. Eberly et al. (MINERvA Collaboration), Phys. Rev. D 92, 092008 (2015).

[16] R. Acciarri et al. (ArgoNeuT Collaboration), Phys. Rev. Lett. 113, 261801 (2014); 114, 039901 (2015).

[17] T. Walton et al. (MINERvA Collaboration), Phys. Rev. D 91, 071301 (2015).

[18] T. Le et al. (for the MINERvA Collaboration), Phys. Lett. B 749, 130 (2015).

[19] K. Abe et al. (T2K Collaboration), Phys. Rev. D 91, 112002 (2015).

[20] M. Martini, M. Ericson, G. Chanfray, and J. Marteau, Phys. Rev. C 80, 065501 (2009).

[21] M. Martini, M. Ericson, G. Chanfray, and J. Marteau, Phys. Rev. C 81, 045502 (2010).

[22] J. E. Amaro et al., Phys. Lett. B 696, 151 (2011).

[23] J. Nieves, I. R. Simo, and M. J. Vicente Vacas, Phys. Rev. C 83, 045501 (2011).

[24] A. Bodek, H. Budd, and M. E. Christy, Eur. Phys. J. C 71, 1726 (2011).

[25] M. Martini, M. Ericson, and G. Chanfray, Phys. Rev. C 84, 055502 (2011).

[26] J. Nieves, I. Ruiz Simo, and M. J. Vicente Vacas, Phys. Lett. B 707, 72 (2012).

[27] J. E. Amaro, M. B. Barbaro, J. A. Caballero, and T. W. Donnelly, Phys. Rev. Lett. 108, 152501 (2012).

[28] O. Lalakulich, K. Gallmeister, and U. Mosel, Phys. Rev. C 86, 014614 (2012).
[29] J. Nieves, I. Ruiz Simo, and M. J. Vicente Vacas, Phys. Lett. B 721, 90 (2013).

[30] M. Martini and M. Ericson, Phys. Rev. C 87, 065501 (2013).

[31] M. Martini and M. Ericson, Phys. Rev. C 90, 025501 (2014).

[32] G. D. Megias, T. W. Donnelly, O. Moreno, C. F. Williamson, J. A. Caballero, R. González-Jiménez, A. De Pace, M. B. Barbaro, W. M. Alberico, M. Nardi, and J. E. Amaro, Phys. Rev. D 91, 073004 (2015)

[33] M. Ericson and M. Martini, Phys. Rev. C 91, 035501 (2015).

[34] J. T. Sobczyk and J. Zmuda, Phys. Rev. C 91, 045501 (2015).

[35] U. Mosel, Phys. Rev. C 91, 065501 (2015).

[36] J. Blietschau et al. (Gargamelle Collaboration), Nucl. Phys. B 133, 205 (1978).

[37] K. Abe et al. (T2K Collaboration), Phys. Rev. Lett. 113, 241803 (2014).

[38] J. Wolcott et al. (MINERvA Collaboration), Phys. Rev. Lett. 116, 081802 (2016).

[39] M. Day and K. S. McFarland, Phys. Rev. D 86, 053003 (2012).

[40] N. Jachowicz, K. Heyde, J. Ryckebusch, and S. Rombouts, Phys. Rev. C 65, 025501 (2002).

[41] V. Pandey, N. Jachowicz, J. Ryckebusch, T. Van Cuyck, and W. Cosyn, Phys. Rev. C 89, 024601 (2014).

[42] V. Pandey, N. Jachowicz, T. Van Cuyck, J. Ryckebusch, and M. Martini, Phys. Rev. C 92, 024606 (2015).

[43] G. Chanfray and P. Schuck, Phys. Rev. A 38, 4832 (1988).

[44] W. M. Alberico, G. Chanfray, J. Delorme, M. Ericson, and A. Molinari, Nucl. Phys. A 634, 233 (1998).

[45] U. Stroth, R. W. Hasse, P. Schuck, W. M. Alberico, A. Molinari, and M. Ericson, Phys. Lett. B 156, 291 (1985).

[46] H. De Vries, C. W. De Jager, and C. De Vries, At. Data Nucl. Data Tables 36, 495 (1987).

[47] J. Delorme, M. Ericson, A. Figureau, and N. Giraud, Phys. Lett. B 89, 327 (1980).

[48] M. Waroquier, J. Sau, K. Heyde, P. Van Isacker, and H. Vincx, Phys. Rev. C 19, 1983 (1979).

[49] J. Ryckebusch, M. Waroquier, K. Heyde, J. Moreau, and D. Ryckbosch, Nucl. Phys. A 476, 237 (1988)

[50] N. Jachowicz, C. Praet, and J. Ryckebusch, Acta Phys. Pol. B 40, 2559 (2009).

[51] M. Ericson and T. E. O. Ericson, Ann. Phys. (NY) 36, 323 (1966).

[52] W. M. Alberico, M. Ericson, and A. Molinari, Nucl. Phys. A 379, 429 (1982).

[53] J. Nieves, J. E. Amaro, and M. Valverde, Phys. Rev. C 70, 055503 (2004); 72, 019902(E) (2005).

[54] K. Abe et al. (T2K Collaboration), Phys. Rev. D 87, 012001 (2013)

[55] A. A. Aguilar-Arevalo et al. (MiniBooNE Collaboration), Phys. Rev. D 79, 072002 (2009).

[56] M. V. Ivanov, G. D. Megias, R. Gonzalez-Jimenez, O. Moreno, M. B. Barbaro, J. A. Caballero, and T. W. Donnelly, J. Phys. G 43, 045101 (2016).

[57] A. Meucci and C. Giusti, Phys. Rev. D 91, 093004 (2015). 